\title{
Collisionless Damping of Laser Wakes in Plasma Channels
}

\author{
G. Shvets and X. Li \\ Princeton Plasma Physics Laboratory, Princeton NJ 08543
}

\begin{abstract}
Excitation of accelerating modes in transversely inhomogeneous plasma channels is considered as an initial value problem. Discrete eigenmodes are supported by plasma channels with sharp density gradients. These eigenmodes are collisionlessly damped as the gradients are smoothed. Using collisionless Landau damping as the analogy, the existence and damping of these "quasi-modes" is studied by constructing and analytically continuing the causal Green's function of wake excitation into the lower half of the complex frequency plane.

Electromagnetic nature of the plasma wakes in the channel makes their excitation nonlocal. This results in the algebraic decay of the fields with time due to phasemixing of plasma oscillations with spatially-varying frequencies. Characteristic decay rate is given by the mixing time $\tau_{m}$, which corresponds to the dephasing of two plasma fluid elements separated by the collisionless skin depth. For wide channels the exact expressions for the field evolution are derived. Implications for electron acceleration in plasma channels are discussed.
\end{abstract}

\section{INTRODUCTION}

Plasma channel is an important tool for a variety of laser-plasma applications, such as laser-driven particle accelerators, $\mathrm{x}$-ray lasers, harmonic generation, and inertial confinement fusion. The primary objective of creating a plasma channel is to guide radiation (e.g. an intense laser pulse in a laser wakefield accelerator) over many Rayleigh lengths. Plasma channels also support low-frequency waves which can be used for particle acceleration. For example, the accelerating wake in the hollow plasma channel is transversely homogeneous and provides a weak linear focusing $[1,2]$.

Theoretical analysis of laser wake excitation in more realistic plasma channels with continuous density profiles is far more challenging, and was first undertaken by Shvets et. al. [2], with the emphasis on the hollow channels with a sharp (although not infinitely sharp) plasma-vacuum interface. In an ideal hollow channel of width $b$ only two plasma modes of oscillation are excited by a short laser pulse: (a) global surface mode with frequency $\omega_{c h}=\omega_{p} / \sqrt{1+k_{p} b}$, which exists in the channel and inside the plasma, and is excited at the plasma-vacuum interface, and (b) 


\section{DISCLAIMER}

This report was prepared as an account of work sponsored by an agency of the United States Government. Neither the United States Government nor any agency thereof, nor any of their employees, make any warranty, express or implied, or assumes any legal liability or responsibility for the accuracy, completeness, or usefulness of any information, apparatus, product, or process disclosed, or represents that its use would not infringe privately owned rights. Reference herein to any specific commercial product, process, or service by trade name, trademark, manufacturer, or otherwise does not necessarily constitute or imply its endorsement, recommendation, or favoring by the United States Government or any agency thereof. The views and opinions of authors expressed herein do not necessarily state or reflect those of the United States Government or any agency thereof. 


\section{DISCLAIMER}

Portions of this document may be illegible in electronic image products. Images are produced from the best available original document. 
electrostatic plasma mode with frequency $\omega_{p}$, which is locally excited in the bulk of the plasma.

In a realistic plasma channel the surface mode turns into a damped quasi-mode, which decays exponentially in time. Qualitatively, a quasi-mode, whose frequency is close to the frequency of the surface mode in a hollow channel [1], is absorbed at the resonant location $x_{r}$, defined by $\omega_{p}\left(x_{r}\right)=\omega_{c h}$. The locally excited plasma wave also decays, but much slower, according to the power law. This occurs because, due to the electromagnetic nature of the plasma wave in a channel, fields at a given spatial location $x$ are affected by the plasma currents within a collisionless skin depth $c / \omega_{p}$ from $x$. Therefore, wakes damp when, roughly, two fluid elements, separated by $c / \omega_{p}$ get out of phase. The detailed analysis of this collisionless damping will appear in an upcoming issue of Physics of Plasmas [3]. Here we outline the general theoretical approach (Section II), present the results of the numerical study of quasi-modes (Section III), and present the analytical results of wake damping in wide plasma channels (Section IV).

\section{THEORETICAL MODEL}

A number of simplifying assumptions make the problem at hand analytically tractable. Slab geometry is assumed, so that the plasma density $n_{0}(x)$ is only a function of the transverse coordinate $x$. Ions are assumed immobile, providing a neutralizing background. Wakes are driven by a non-evolving nonrelativistic laser pulse with $a=e|A| / m c^{2}<1$, so that all calculations are performed to order $a^{2}$. The group velocity of the pulse is assumed close to $c$ (tenuous plasma), and all the plasma quantities are functions of $\zeta=t-z / c$.

In a recent work [2] we demonstrated that the Laplace transformed electric field of the wake is

$$
\epsilon \overrightarrow{\tilde{E}}=\frac{\omega_{p 0}^{2}(x)}{\omega^{2}} \vec{\nabla} \tilde{f}+i \frac{c}{\omega} \nabla \times \overrightarrow{\tilde{B}}
$$

where $f=-m c^{2}|\vec{a}|^{2} / 4 \mathrm{e}$ is the ponderomotive potential and $\epsilon(x, \omega)=1-\omega_{p 0}^{2}(x) / \omega^{2}$. All Laplace transformed variables are "tilded". Magnetic field is calculated by substituting Eq. (1) into Faraday's law: $\mathcal{L}_{S}(\omega) \tilde{B}_{y}=\omega^{2} \epsilon^{\prime} \tilde{f} / c^{2} \epsilon^{2}$, where $\mathcal{L}_{\text {S }}$ is a SturmLouville operator, parametrically dependent on the complex frequency $\omega$ :

$$
\mathcal{L}_{S}(\omega)=\frac{d}{d x}\left(\frac{1}{\epsilon(x, \omega)} \frac{d}{d x}\right)-\frac{\omega_{p}^{2}(x)}{c^{2} \epsilon(x, \omega)} .
$$

Magnetic field in $\zeta$ - domain is then obtained by inverse Laplace transforming $\tilde{B}_{y}(x, \omega)$ along any contour $\Gamma$ in the upper half plane of complex $\omega$, as shown in Fig. 1a. Since we only consider accelerating TM modes, boundary conditions $\tilde{B}_{y}(x=0)=\tilde{B}_{y}(x=\infty)=0$ must be satisfied. Assuming a monotonic density plasma density profile, which asymptotes to $n_{0}$ at infinity, one can choose a 
transverse location $x_{\max }$ (finite or infinite), such that $\omega_{p}(x) \equiv \omega_{p 0}$ for $x>x_{\max }$. Therefore, the second boundary condition can be chosen at $x_{\max }$ as $\tilde{B}^{\prime}+k_{p 0} B=0$.

Magnetic field $\tilde{B}_{y}$ is computed by constructing the Green's function $G\left(x, x^{\prime}, \omega\right)$ of the operator $\mathcal{L}_{S}$, satisfying the boundary conditions at $x=0$ and $x=x_{\max }$, and a differential equation $\mathcal{L}_{S} G\left(x, x^{\prime}, \omega\right)=\delta\left(x-x^{\prime}\right)$. Such approach was originally used by Briggs et. al. to study the eigenmodes of a strongly magnetized noneutral plasma [4]. By causality, we require that $G\left(x, x^{\prime}, \omega\right)$ has no singularities in the upper half $\omega$ - plane.

The Green's function of $\mathcal{L}_{S}$ is

$$
G\left(x, x^{\prime}, \omega\right)=\frac{1}{D(\omega)} \begin{cases}\phi_{+}(x, \omega) \phi_{-}\left(x^{\prime}, \omega\right) & \text { for } x^{\prime}<x \\ \phi_{-}(x, \omega) \phi_{+}\left(x^{\prime}, \omega\right) & \text { for } x^{\prime}>x\end{cases}
$$

where $\phi_{-}$and $\phi_{+}$are linearly independent solutions of $\mathcal{L}_{S} \phi_{ \pm}=0$, satisfying their respective boundary conditions: $\phi_{-}(x=0, \omega)=0$ and $\phi_{+}^{\prime}\left(x=x_{\max }, \omega\right) / \phi_{+}(x=$ $\left.x_{m a x}, \omega\right)=-\omega_{p 2} / c$. Constant $D(\omega)$ is the Wronskian of $\phi_{ \pm}$, given by $D(\omega)=$ $\left[\phi_{+}^{\prime} \phi_{-}-\phi_{-}^{\prime} \phi_{+}\right] / \epsilon . \quad D(\omega)$ will be referred to as a dispersion function because its zeros correspond to the eigenmodes of a plasma channel. For example, for an ideal hollow channel $D\left(\omega_{c h}\right)=0$. Incidentally, for a plasma channel with continuous density profile dispersion function never vanishes. Therefore, realistic channels do not support any continuous eigenmodes. A rigorous proof is given in [3].

We can now formally solve for the magnetic field:

$$
\tilde{B}_{y}(x, \omega)=\int_{0}^{x_{\max }} d x^{\prime} G\left(x, x^{\prime}, \omega\right)\left(\frac{\omega^{2}}{c^{2}} \frac{\epsilon^{\prime}}{\epsilon^{2}} \tilde{f}\left(x^{\prime}, \omega\right)\right)
$$

Similar Green's functions can be derived for the electric field.
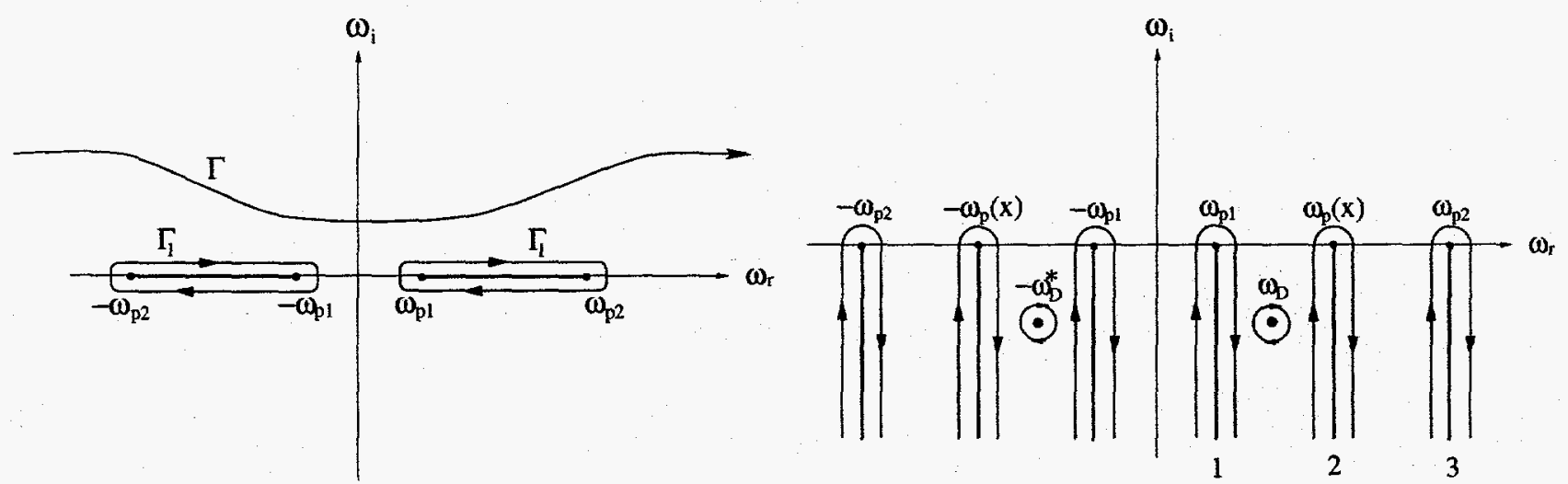

FIGURE 1. (a) $\Gamma$ is integration path for the inverse Laplace transform). Integral along the contour $\Gamma$ can be reduced to a sum of two integrals along branch cuts (contours $\Gamma_{1}$ ). (b) A different cut which recovers the quasi-modes $\omega_{D}$ and $\omega_{D}^{*}$-zeros of the analytic continuation of the dispersion function $D_{*}(\omega)$. 
For $\omega_{i}>0$ the eigenfunctions $\phi_{-}$and $\phi_{+}$can be expressed in terms of any two linearly independent solutions of the homogeneous equation $\mathcal{L}_{S} \phi=0$. One example of such functions is a set $\left(\phi_{0}, \phi_{1}\right)$, satisfying $\phi_{0}(0)=0, \phi_{0}^{\prime}(0)=1$, and $\phi_{1}(0)=\epsilon(x=0, \omega), \phi_{1}^{\prime}(0)=0$. Integrating these solutions according to $\mathcal{L}_{S} \phi_{0,1}=0$ between $x=0$ to $x=x_{m a x}$ yields the dispersion function $D(\omega)$, which is given by

$$
D=\frac{\phi_{0}^{\prime}\left(x_{\max }\right)+k_{p 2} \phi_{0}\left(x_{\max }\right)}{\phi_{1}^{\prime}\left(x_{\max }\right)+k_{p 2} \phi_{1}\left(x_{\max }\right)},
$$

and the $\phi_{ \pm}$basis functions: $\phi_{-}=\phi_{0}$ and $\phi_{-}=\phi_{0}-D(\omega) \phi_{1}$. Integration of the homogeneous equation along the real axis is unambiguous for $\omega_{i}>0$ since the singularities of $\mathcal{L}_{S}$ lie above the real $x$-axis.

Singularities of $\mathcal{L}_{S}$ are encountered when $\omega$ assumes a real value from the interval $\left[\omega_{p 1}, \omega_{p 2}\right]$. The dispersion function $D(\omega)$ thus has branching points at $\omega_{p 1}$ and $\omega_{p 2}$. Basis functions $\phi_{ \pm}(x, \omega)$ have another branching point at $\omega=\omega_{p}(x)$. One can insure that $\tilde{B}_{y}(x, \omega)$ is single-valued by making a single cut between $\omega_{p 1}$ and $\omega_{p 2}$.

To recover the damped quasi-modes a different cut can be used (following Ref. [4]), as shown in Fig. 1b, where the integration contour is pushed into the lower half plane. To do that, $D(\omega), \phi_{-}(x, \omega)$, and $\phi_{+}(x, \omega)$ have to be analytically continued for $\omega_{i}<0$. Although $D(\omega)$ has no zeros above or below the real $\omega$ axis, its analytic continuation $D_{*}(\omega)$ may have zeros $\omega_{D}$ and $-\omega_{D}^{*}$ in the lower half-plane, as shown in Fig. 1b. This phenomenon is analogous to the collisionless Landau damping of electrostatic plasma waves, where the dielectric constant does not vanish for any complex frequency while its analytic continuation does vanish, resulting in Landau-damped quasi-modes.

With $\omega_{i}>0$, the complex resonant point $x_{r}(\omega)$, defined by $\epsilon\left(\omega, x_{r}\right)=0$, is located in the upper half of the $x$ - plane. Basis functions $\phi_{0,1}$ can be integrated along any contour which connects the origin and $x$ (including the straight line, of course), provided that the contour stays below the resonant point $x_{r}(\omega)$, as shown in Fig. 2a. When $\omega$ is moved into the lower half-plane, the resonant point $x=x_{r}(\omega)$ is also lowered below the real axis. Therefore, to ensure analyticity of $\phi_{0,1}(x, \omega)$ as a function of $\omega$ the integration contour in the complex $x-$ plane must be deformed to stay below the branching point $x=x_{r}(\omega)$, as shown in Fig. 2b.

For a given $\omega$ we have to choose the branch cut in the $x$ - plane, corresponding to the cuts in the $\omega$ plane, shown in Fig. 1a. It can be shown [3] that this cut intersects the real $x-$ axis at a point $x_{d}$, such that $\omega_{p}\left(x_{d}\right)=\operatorname{Re}(\omega)$. Basis functions $\phi_{ \pm}(x, \omega)$ are discontinuous at $x_{d}$. As was pointed out by Briggs [4], discontinuity of the field is not "real" in the sense that it only exists in frequency domain and disappears after integration over $\omega$.

With the procedures for the analytic continuation of the basis functions and the dispersion function $D(\omega)$ rigorously established, the inverse Laplace transform integration can be carried out along the branch cuts and around the poles of the analytically continued Green's function, as shown in Fig. 1b. Zeros of the analytically continued dispersion function $\left(\omega_{D},-\omega_{D}^{*}\right)$ define the collisionlessly damped quasimodes of the plasma channel. Note that since the quasi-modes are discontinuous 
at $x_{d}\left(\omega_{D}\right)$, their existence does not contradict the earlier statement that realistic channels do not support continuous eigenmodes. Contributions of the quasi-modes are important for the short-time evolution of the plasma wakes, $\zeta<1 / \operatorname{Im}\left(\omega_{D}\right)$. For longer times quasi-modes damp out, and branch cut contributions dominate. This is because the fields associated with the branch cuts algebraically decay in $\zeta$. Moreover, for very wide plasma channels quasi-modes are important in a very small part of the channel, and the branch cut 2 dominates throughout the rest of the channel. In the rest of the paper we separately consider the global quasi-modes and the local plasma excitations, associated with the branch cut 2.

\section{WEAKLY DAMPED QUASI-MODES}

Undamped electromagnetic surface mode, supported by an ideal hollow channel with an infinitely sharp vacuum-plasma interface, becomes a weakly damped quasi-mode as the plasma-vacuum interface is smoothed out. For a channel with continuous plasma density a resonant location $x_{d}$ exists, where the surface mode frequency is equal to the local plasma frequency. As a result of the coupling between the surface mode and the electrostatic plasma wave, the former damps. Quasi-mode is peaked at and exponentially decays away from the resonance point $x_{d}$. Quasimode is distinct from the localized electrostatic plasma waves in that it is global (i. e. exists throughout the channel), yet possess a well-defined frequency which is independent of the transverse location $x$. It is not, however, a true eigenmode: it belongs to the continuum of the plasma waves. Weakly damped quasi-modes are
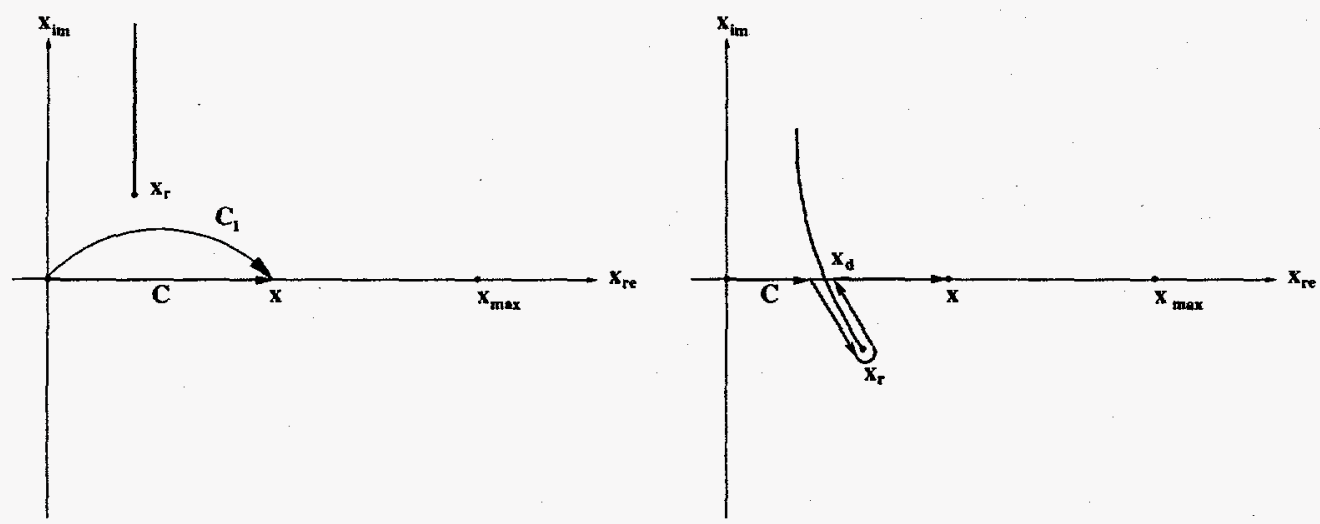

FIGURE 2. Integration paths in the complex $x$ plane of the differential equation $\mathcal{L}_{S}(\omega) \phi_{0,1}=0$ for the basis functions $\phi_{0}$ and $\phi_{1}$, whose boundary conditions are set at $x=0 . x_{r}$ is the resonance point, defined by $\epsilon\left(x_{r}, \omega\right)=0$. (a) $\omega$ in the upper half plane, $\operatorname{Im} \omega>0 . C_{1}$ and $C$-possible integration paths. Basis functions are continuous on the real axis. (b) $\omega$ in the lower half plane, $\operatorname{Im} \omega<0$. Integration contour remains below the resonance point $x_{r}$ to ensure analytic continuation of the Green's function. Basis functions are discontinuous on the real axis at $x=x_{d}$, where $\epsilon\left(x_{d}, \operatorname{Re} \omega\right)=0$. 
important only for short times of order the damping time, after which the coherent, single-frequency motion of the plasma electrons is destroyed, and the local fields, oscillating with the local plasma frequencies $\omega_{p}(x)$, prevail.

As an example, consider the parabolic plasma channels

$$
\omega_{p}^{2}(x)=\omega_{p 1}^{2}+\frac{\left(\omega_{p 0}^{2}-\omega_{p 1}^{2}\right) x^{2}}{x^{2}+b^{2}}
$$

of two types: (a) hollow on axis $\left(\omega_{p 1}=0\right)$, or (b) with finite on-axis density $\left(\omega_{p 1} \neq 0\right)$. For such chanels $D_{*}(\omega)$ is found from Eq. (5), where the basis functions $\phi_{0,1}$ are numerically integrated from $x=0$ to $x=x_{\text {max }}$ along the contour shown in Fig. 2b. In practice, the integration contour was taken as a sum of two straight lines in the complex plane: one, connecting $x=0$ and $x=x_{r}(\omega)-i \nu$ (where $\nu$ is an arbitrary positive number) and the other, connecting $x=x_{r}(\omega)-i \nu$ and $x_{\max }$ (chosen at $x_{\max }=3 b$ ). The real frequency and the damping rate of the quasi-modes of are plotted as functions of the dimensionless channel width $k_{p 0} b$ in Figs. 3a,b.

For a hollow channel the only contribution to the accelerating gradient $E_{z}(x=0)$ comes from the quasi-mode. A fluid code, which calculates all the electromagnetic fields and plasma fluid quantities in the $\zeta$ domain was recently developed [5]. To extract the damping coefficient and the frequency from the fluid code, we fitted $E_{z}(x=0, \zeta)$ by $E_{0} \cos \left(\omega_{r} \zeta+\phi_{0}\right) \exp (-\gamma \zeta)$, where $E_{0}$ and $\phi_{0}$ are constants. The results of the fit are shown in Fig. 4, where a narrow plasma channel $k_{p 0} b=1$ was simulated. Both the damping rate and the frequency exactly correspond to those of the eigenmode $\left(k_{p 0} b=1\right.$ point of the plots in Fig. 3a).

(a) $\omega_{\mathrm{pl}}=0$

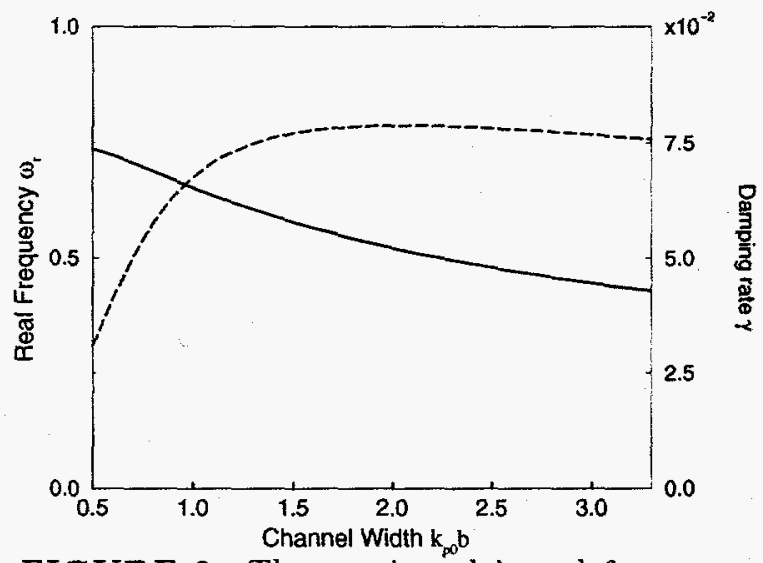

(b) $\omega_{\mathrm{pl}}=0.8 \omega_{\mathrm{p} 0}$

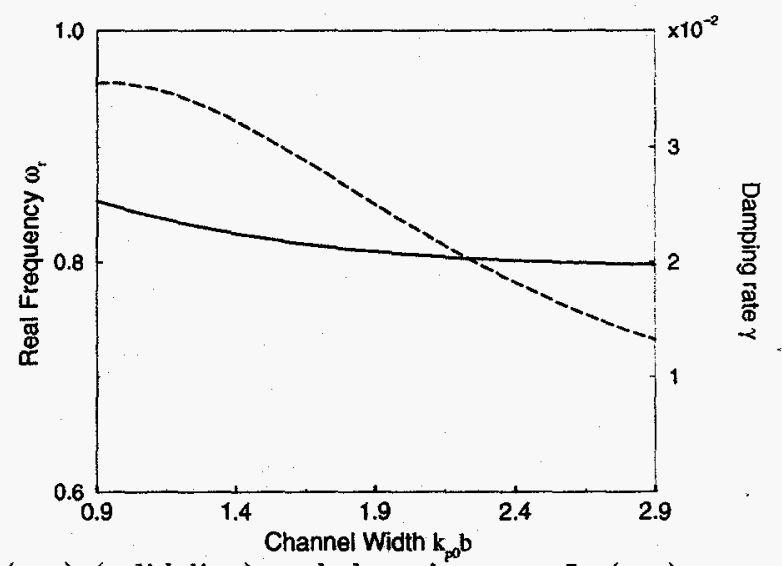

FIGURE 3. The quasi-mode's real frequency $\operatorname{Re}\left(\omega_{D}\right)$ (solid line) and damping rate $\operatorname{Im}\left(\omega_{D}\right)$ (dashed line) as a function of the channel width $k_{p 0} b$ for two types of plasma channels: (a) zero plasma density on axis and (b) finite plasma density on axis. Plasma frequency satisfies $\omega_{p}^{2}(x)=\omega_{p 1}^{2}+\left(\omega_{p 0}^{2}-\omega_{p 1}^{2}\right) x^{2} / x^{2}+b^{2}$. 


\section{WAKE DAMPING IN WIDE CHANNELS}

Contributions of the quasi-modes are not sufficient to describe the electromagnetic wakes for all times and at all transverse locations inside the plasma channel. The quasi-mode contribution to the total electromagnetic fields at a given transverse point can be negligible if the quasi-mode is localized far away from that location. For example, the quasi-mode of a wide channel becomes exponentially small sufficiently far away from the resonance point. Figure 5 shows the spatial profiles of the accelerating and focusing electric fields, corresponding to the quasimode of a channel, described by Eq. (6) with $k_{p 0} b=3$ and $\omega_{p 1}=0$. Both fields peak at $x_{d}\left(\omega_{D}\right) \approx 1.5 / k_{p 0}$, where $\omega_{D}=\omega_{p 0}(0.45-0.077 i)$ is the complex frequency of the quasi-mode. Note that the accelerating field, corresponding to the quasi-mode, is discontinuous at $x_{d}$. As explained earlier, this does not correspond to a physical discontinuity. The values of both fields at the edge of the channel $(x=2 b)$ are negligible in comparison with their peak values at $x=x_{d}$. Therefore, EM fields far from $x_{d}$ are dominated by local excitations. The evolution of these excitations, described by the branch cut 2 , is computed below for wide channels.

We assume that the scale of the plasma inhomogeneity is much longer than the skin depth: $k_{p} L \gg 1$, where $L=k_{p} / k_{p}^{\prime}$. The laser profile is also assumed only weakly nonuniform on a scale $1 / k_{p}: \partial \ln |f| / \partial x \ll k_{p}$. The exact analytic expressions for the $\phi_{+}$and $\phi_{-}$can be obtained, enabling the exact integration of the Green's function in $x^{\prime}$ [see Eq. (4)] and $\omega$ (inverse Laplace transform).

In the vicinity of the resonant point $\left|x-x_{r}\right| \ll L$ the lowest-order Taylor expansion for the plasma dielectric function can be used: $\epsilon=\epsilon^{\prime}\left(x-x_{r}\right)$. Therefore, in the vicinity of $x_{r}$ basis functions $\phi_{ \pm}$satisfy $\phi^{\prime \prime}-\phi^{\prime} /\left(x-x_{r}\right)-k_{p}^{2} \phi=0$. We further assume that $x$ is at least $1 / k_{p}$ away from both the origin and the discontinuity point $x_{d}\left(\omega_{D}\right)$. The origin can now be treated as being at $-\infty$. The basis functions are $\phi_{-}=t K_{1}(t)+i \pi t I_{1}(t)$ and $\phi_{+}=t K_{1}(t)$, where $t=k_{p}\left(x-x_{r}\right)$, and $I_{1}(t)$ and $K_{1}(t)$ are the modified Bessel functions.

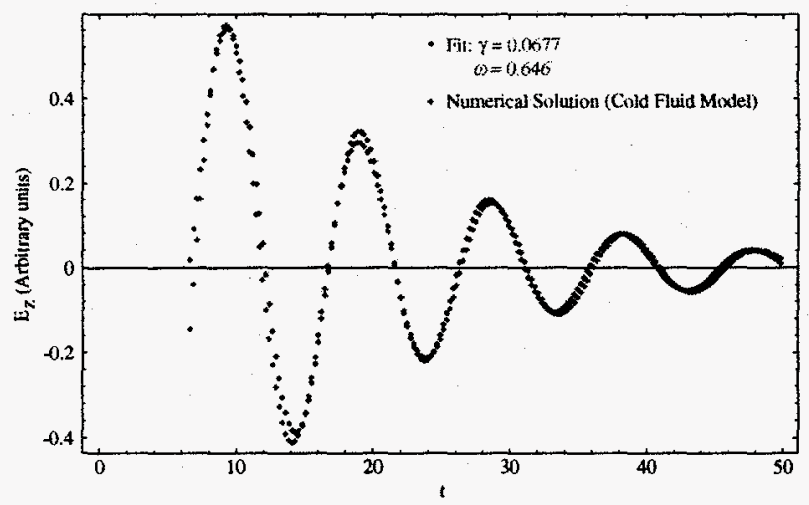

FIGURE 4. Numerical fit of the on-axis accelerating field from a fluid simulation by the formula $E_{z}(x=0, \zeta)=E_{0} \cos \left(\omega_{r} \zeta+\phi_{0}\right) \exp (-\gamma \zeta)$. 


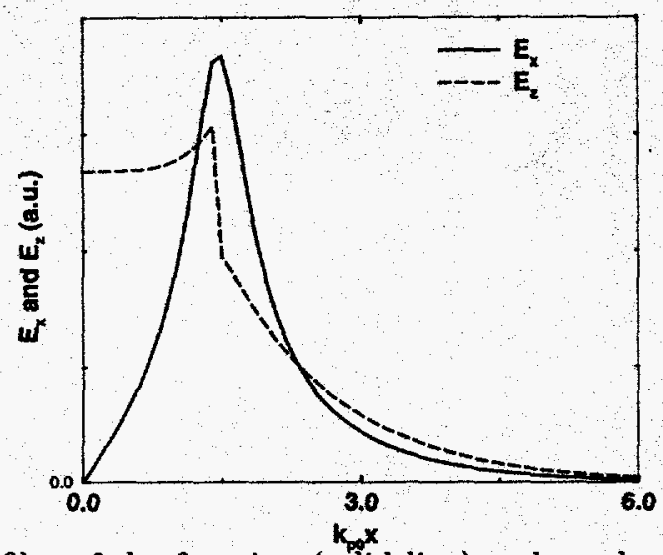

FIGURE 5. Spatial profiles of the focusing (solid line) and accelerating (dashed line) electric fields of the collisionlessly damped quasi-mode of a wide plasma channel. Plasma frequency satisfies $\omega_{p}^{2}(x)=\omega_{p 0}^{2} x^{2} / x^{2}+b^{2}$; channel width $k_{p 0} b=3$. Accelerating field $E_{z}$ of the quasi-mode is discontinuous at $x_{d} \approx 1.5 k_{p 0}^{-1}$.

The details of the integrations in $x^{\prime}$ and in $\nu$ (which parametrizes the branch cut 2: $\left.\omega=\omega_{p}(x)-i \nu\right)$ are given elsewhere [3]. Here we only present the results:

$$
\begin{aligned}
& E_{x}(x, \zeta)=-\sin \left(\omega_{p} \zeta\right) \omega_{p} \frac{\partial \tilde{f}\left(x, \omega_{p}\right)}{\partial x}\left[1+\frac{\zeta^{2}}{\tau_{m}^{2}}\right]^{-\frac{3}{2}}-\cos \left(\omega_{p} \zeta\right) \frac{\omega_{p}^{2}}{c} \tilde{f}\left(x, \omega_{p}\right) \frac{\zeta / \tau_{m}}{\sqrt{1+\zeta^{2} / \tau_{m}^{2}}} \\
& E_{z}(x, \zeta)=\cos \left(\omega_{p} \zeta\right) \frac{\omega_{p}^{2}}{c} \tilde{f}\left(x, \omega_{p}\right) \frac{1}{\sqrt{1+\zeta^{2} / \tau_{m}^{2}}} \\
& B_{y}(x, \zeta)=-2 \sin \left(\omega_{p} \zeta\right) \frac{k_{p}}{\tau_{m}} \tilde{f}\left(x, \omega_{p}\right)\left[1+\frac{\zeta^{2}}{\tau_{m}^{2}}\right]^{-\frac{3}{2}}
\end{aligned}
$$

where $\omega_{p} \equiv \omega_{p}(x)$ is the local plasma frequency at the transverse position $x$. In deriving Eqs. $(7,8,9)$ the second and higher derivatives of $f$ are neglected.

In a homogeneous plasma the second term in Eq. (7) identically vanishes. Interestingly, in a channel it comes to dominate the locally excited transverse electric field (described by the first term) for $\zeta \gg \tau_{m}$. Also note that this non-decaying component of the focusing electric field is in phase with the accelerating field. This is potentially important for plasma-based particle accelerators. In a standard laser wakefield accelerator, based on a transversely uniform plasma, the accelerating and focusing electric fields are 90 degrees out of phase. Therefore, the phase region, where an injected particle is both accelerated and focused, is only $\lambda_{p} / 4$ long. In a channel this region can be $\lambda_{p} / 2$ long, as seen from Eqs. $(7,8)$.

Equations $(7,8,9)$ constitute an important result of this paper. For the first time, the exact expressions for the wake evolution in wide plasma channels, valid for 
arbitrary times, have been derived, and the phase-mixing time introduced. The physical meaning of the phase-mixing in plasma channels is explained below.

Damping of the electromagnetic fields in plasma channels is reminiscent of the decay of two-dimensional plasma oscillations due to the trajectories crossing, as described by Dawson [6]. To appreciate the differences between these two damping mechanisms, consider the underlying basic physics. Local plasma oscillations (and the corresponding electric field) decay when different plasma fluid elements, oscillating with their local plasma frequencies, get out of phase. Consider two fluid elements, initially located at $x=x_{0}$ and $x=x_{0}+\Delta x$ and oscillating with their local plasma frequencies $\omega_{p}\left(x_{0}\right)$ and $\omega_{p}\left(x_{0}+\Delta x\right)$, respectively. These two elements get out of phase after $\tau=\left|\omega_{p}^{\prime} \Delta x\right|^{-1}$.

Electrostatic cold plasma oscillations, considered by Dawson, collapse when adjacent fluid elements collide with each other. This is because there is no Pointing flux associated with such oscillations, so the energy can only be physically carried by the plasma electrons. This trajectory crossing can be interpreted as dephasing between the electrons separated by the distance equal to the amplitude of their oscillation $A$. Therefore, the nonlinear wave breaking time $\tau_{W B}=\left|A \omega_{p}^{\prime}\right|^{-1}$ is calculated by taking $\Delta x=A$. For very small oscillation amplitudes this transverse wave breaking takes place several plasma periods behind the laser pulse [7].

On the other hand, wakes in plasma channels have a non-vanishing Pointing flux, enabling communication between different transverse positions. As a result, electromagnetic wakes in channels become nonlocal: fields at a given spatial location $x$ are affected by the plasma currents within a collisionless skin depth $c / \omega_{p}$ from $x$. Therefore; wakes damp when, roughly, two fluid elements, separated by $c / \omega_{p}$ get out of phase, that is, $\Delta x=c / \omega_{p}$, or $\tau_{m}=\omega_{p} / c \omega_{p}^{\prime}$. Below we demonstrate how this estimate can be obtained in a more formal way.

As Eqs. $(7,8,9)$ indicate, magnetic field decays faster than both components of the electric field. Due to the conservation of the total vorticity $(\nabla \times \vec{v}=e \vec{B} / m c)$, plasma flow becomes almost curl-free for large times: $\partial v_{z} / \partial x=\partial v_{x} / \partial z$, or $v_{z}=$ $-v_{x} \tau_{m} / \zeta$. Combining Maxwell's equations with the vorticity conservation yields

$$
\left[\frac{\partial^{2}}{\partial x^{2}}-k_{p}^{2}(x)\right] B_{y} \approx \frac{4 \pi e n_{0}}{c^{2} \zeta} \hat{v}_{x} e^{-i \omega_{p}(x) \zeta}+\text { c. c. }
$$

where we have used the curl-free flow assumption and the fact that, for large $\zeta, v_{x}$ oscillates with a constant amplitude. Equation (10) can be solved in closed forms by expanding $\omega_{p}\left(x^{\prime}\right) \approx \omega_{p}(x)+\omega_{p}^{\prime}\left(x^{\prime}-x\right)$ :

$$
B_{y}(x, \zeta)=-\frac{2 \pi e n_{0} \hat{v}_{x}}{k_{p} c^{2} \zeta} e^{-i \omega_{p}(x) \zeta} \int_{-\infty}^{+\infty} d x^{\prime} e^{-k_{p}\left|x-x^{\prime}\right|} e^{i \omega_{p}^{\prime} \zeta\left(x-x^{\prime}\right)}+\text { c. c. }
$$

As illustrated by the integrand of Eq. (11), fluid elements within one collisionless skin depth from $x$ contribute to $B_{y}(x)$. For large $\zeta$ these contributions get out of phase, and the integral in $\mathrm{Eq}$. (11) decays as $\left(1+\zeta^{2} / \tau_{m}^{2}\right)^{-1}$. Therefore, magnetic 
field decays as $\zeta^{-3}$, in agreement with Eq. (9). This calculation illustrates how the electromagnetic nature of the channel wake results in the nonlocal excitation on a $c / \omega_{p}$ scale, leading to phase-mixing of the plasma fluid and algebraic decay of the wake.

If the plasma oscillation amplitude $A$ is much smaller than the collisionless skin depth, phase mixing occurs before the wave breaking. Transverse wave breaking can still take place after $\tau_{W B} \gg \tau_{m}$, which can be estimated by requiring that $\left|\partial_{x} E_{x}\right|=4 \pi e n_{0}$. Assuming a short laser driver with duration of order $1 / \omega_{p}$ and nonrelativistic normalized vector potential $\left|\vec{a}^{2}\right| \ll 1$, estimate that $\tau_{W B} \approx 2 \tau_{m} /\left|\vec{a}^{2}\right|$.

\section{CONCLUSIONS}

In summary, the highlights of the theory of collisionless damping of laser wakes in plasma channels is presented. Two types of decaying solutions are identified: exponentially decaying global quasi-modes and algebraically decaying almost-local excitations. Frequencies and damping rates of the quasi-modes for various channels are found, in excellent agreement with the results of the explicit fluid simulations. Almost-local wake fields decay because of the phase-mixing of the plasma currents at different transverse locations inside the channel.

\section{ACKNOWLEDGMENTS}

This work was supported by the US DOE Division of High Energy Physics. The authors acknowledge useful conversations with N. J. Fisch, B. A. Shadwick, and J. S. Wurtele.

\section{REFERENCES}

1. T. C. Chiou et. al., Phys. Plasmas 2, 310 (1995).

2. G. Shvets et. al., IEEE Trans. Plasma Sci. 24, 351 (1996).

3. G. Shvets and X. Li, "Theory of Laser Wakes in Plasma Channels", submitted to Phys. Plasmas (1998).

4. R. J. Briggs, J. D. Daugherty, and R. H. Levy, Phys. Fluids 13, 421 (1970).

5. B. A. Shadwick, J. S. Wurtele, "Numerical simulation of wake generation in plasma channels", in preparation (1997).

6. J. M. Dawson, Phys. Rev. , 113, 383 (1959).

7. S. V. Bulanov et. al., Phys. Rev. Lett., 78, 4205 (1997). 